\title{
Numerical Investigation for Aerodynamic Derivatives of Bridge Deck Using DES
}

\author{
Liu Ketong* and Tang Aiping
}

School of Civil Engineering, Harbin Institute of Technology, Harbin, 150090, P.R. China

\begin{abstract}
Detached Eddy Simulation (DES) is quite a new approach for the treatment of turbulence, which unites the efficiency of Reynolds Averaged Navier-Stokes Simulation (RANS) and the accuracy of Large Eddy Simulation (LES) into one framework. In this paper, DES method based on Spalart-Allmaras (S-A) turbulence model is employed to simulate the incompressible viscous flow around bridge decks. In order to obtain the aerodynamic forces, the forced motion simulations of the bridge decks are implemented by self-developed codes combined with FLUENT software. After obtaining the aerodynamic forces, aerodynamic derivatives are determined based on the least square algorithm. As the examples, the thin flat plate and the Great Belt East Bridge suspended spans cross-section are investigated to calculate their aerodynamic derivatives. Finally, the simulation results are compared to the data reported in other studies. The comparisons show that the present method gives much better prediction of the aerodynamic derivatives than RANS method and discrete vortex method (DVM).
\end{abstract}

Keywords: bridge decks, detached eddy simulation, large eddy simulation, the least square algorithm, turbulence model.

\section{INTRODUCTION}

It is very important for bridge flutter prediction to obtain the accurate aerodynamic derivatives, which are important basic parameters for bridge flutter stability analysis. Although wind tunnel tests are the main means to obtain the bridge dynamic derivatives at present, they are costly, timeconsuming, poor repeatability and difficult to effectively study the evolution of the flow field around the bluff body. In recent years, the computational fluid dynamics method attracted people's attention as an effective method and applied to bridge wind engineering. Walther and Larsen [13] used discrete vortex method (DVM) to simulate the flow field around bridge deck, and calculated aerodynamic derivatives for five typical bridge section. Based on large eddy simulation, Selvam et al. [4] employed finite difference method (FDM) and finite element method (FEM) to calculate the drag coefficients and Strouhal numbers for Great Belt East Bridge. They found that the calculation results by FDM are in good agreement with the experimental results. Frandsen et al. [5, 6] applied FEM to explore the flow field around Great Belt East Bridge and to calculate aerodynamic derivatives, and the computed results showed good agreement with those from wind tunnel tests. Based on finite volume method software FLUENT and LES, Sarwar $e t$ al. [7] studied the evolution of the flow field around box girder section by a three-dimensional model. They found that geometric details of girder sections will cause a certain impact on the aerodynamic parameters. Huang et al. [8]

*Address correspondence to this author at the School of Civil Engineering, Harbin Institute of Technology, Harbin, 150090, P. R. China;

Tel: 15945688291; Fax: 0451-86282704; E-mail: ketong-1982@163.com computed aerodynamic derivatives for flat plate and long span bridge deck by FLUENT combined with two-equation $k-\varepsilon$ renormalization group (RNG) turbulence model. Braun et al. [9] developed a FEM model to simulate wind action over the Guamá River Bridge employing the pseudocompressibility approach, large eddy simulation (LES), an arbitrary Lagrangean-Eulerian formulation and an explicit two-step Taylor-Galerkin method. The aerodynamic behavior of the Great Belt East Bridge is also predicted in their research. Other researchers, such as Cao $[10,11]$, Zhu [12-14], and Zhou et al. [15], also have done a lot of research using different simulation methods for this subject.

Since the wind field is typical high Reynolds flow, the existing studies usually adopt RANS or LES to simulate the air flow around bridges. In practice, some complex phenomena such as flow separation, reattachment and trailing vortex shedding unsteadiness always take place in the flow around bridge decks. Some turbulence models of the RANS method have difficulties in simulating flow separation, reattachment and flow recovery around blunt bodies. In addition, the RANS method provides poor description of the flow physics because it is unable to capture effectively the spectrum of unsteadiness that are associated with the turbulent fluctuations away from the wall [16]. All these reasons will affect the calculation accuracy of the RANS method. LES has high simulation accuracy. However, it requires very fine grid to capture the complex flow phenomena in near-wall region. The RANS method is relatively mature in the treatment of near-wall region, and is more skillful in near-wall predictions than LES. It is more important that its predicting results for near-wall flows can meet with the real flow state well. Hence, the hybrid RANS/LES idea of combining both methods where the inner 
near-wall region is handled by the RANS approach while the outer region of the bulk flow is solved through LES makes rather perfect sense. This is the basic idea of DES. S-A turbulence model [17] is the most successful one-equation model and is skilled in predicting flows with strong adverse pressure gradients near the wall boundary. Moreover, it is worth-mentioning here that it can get better results without very fine grid and even can outperform some two-equation turbulence models in predictions of flow separation and reattachment. For such reasons, the S-A model is selected to be incorporated into LES for simulating turbulent flows around bridge decks in the present study. In order to obtain the aerodynamic forces, the forced motion simulations of the bridge decks are implemented by self-developed codes combined with FLUENT software. Numerical simulations of the aerodynamic derivatives for the thin flat plate and Great Belt East Bridge are carried out, and the simulation results are compared against data reported in other studies [18-20] to demonstrate the usefulness and effectiveness of this present work.

\section{NUMERICAL METHODS}

\subsection{Identification Method for Aerodynamic Derivatives}

The aerodynamic forces acting on a bridge deck per unit length can be formulated in the Scanlan and Tomko linear form, which involves eight aerodynamic derivatives:

$$
\begin{aligned}
& L=\rho U^{2} B\left[K H_{1}^{*} \frac{\dot{h}}{U}+K H_{2}^{*} \frac{B \dot{\alpha}}{U}+K^{2} H_{3}^{*} \alpha+K^{2} H_{4}^{*} \frac{h}{B}\right] \\
& M=\rho U^{2} B\left[K A_{1}^{*} \frac{\dot{h}}{U}+K A_{2}^{*} \frac{B \dot{\alpha}}{U}+K^{2} A_{3}^{*} \alpha+K^{2} A_{4}^{*} \frac{h}{B}\right]
\end{aligned}
$$

where $L$ and $M$ are the lift force and the pitching moment, $\rho$ is the air density, $U$ is the inflow wind speed, $B$ is the breadth of the bridge, $H_{i}^{*}$ and $A_{i}^{*}(i=1-4)$ are defined as aerodynamic derivatives, $K=\omega B / U$ is the reduced frequency, $\omega$ is the circular frequency of oscillation, $h$ and $\dot{h}$ are the vertical motion and its time derivatives respectively, $\alpha$ and $\dot{\alpha}$ are the section torsion and corresponding time derivatives, respectively.

In order to identify the eight aerodynamic derivatives in Eq. (1), the bridge section is deemed to be a rigid body and forced to oscillate vertically or to oscillate torsionally in a sinusoidal manner in the flow. The pressure and the friction on the bridge surface can be calculated by solving the governing equations among the computational domain. Then, the aerodynamic forces (the lift force and the pitching moment) can be obtained through surface integral for the pressure and the friction. When the aerodynamic forces are obtained, the aerodynamic derivatives can be extracted by least squares method.

Taking vertical forced vibration as an example explains how to identify aerodynamic derivatives. Assuming the bridge deck is forced to oscillate vertically in the following manner:

$h(t)=h_{0} \sin \omega t$ where $h_{0}$ and $\omega$ are the displacement amplitude and the circular frequency of the oscillation, respectively. The velocity of the bridge deck $\dot{h}(t)$ can be easily obtained from Eq.(2). $h(t), \dot{h}(t)$ and the simulated aerodynamic forces are saved as the discrete data and denoted as $h_{i}, \dot{h}_{i}, L_{i}, M_{i}$ $(i=1,2, \mathrm{~K}, N), N$ is the total recording points. Because only $H_{1}^{*}, H_{4}^{*}, A_{1}^{*}$ and $A_{4}^{*}$ are related with vertical oscillation, Eq.(1) degenerates into the following form:

$$
\begin{aligned}
& L=\rho U^{2} B\left(K H_{1}^{*} \frac{\dot{h}}{B}+K^{2} H_{4}^{*} \frac{h}{B}\right) \\
& M=\rho U^{2} B\left(K A_{1}^{*} \frac{\dot{h}}{B}+K^{2} A_{4}^{*} \frac{h}{B}\right)
\end{aligned}
$$

At each time point $i$, the residuals of the simulated aerodynamic forces and flutter derivatives computed by Eq. (3) can be calculated as follows:

$$
\begin{aligned}
& \delta(L)_{i}=L_{i}-\rho U^{2} B\left(K H_{1}^{*} \frac{\dot{h}}{B}+K^{2} H_{4}^{*} \frac{h}{B}\right) \\
& \delta(M)_{i}=M_{i}-\rho U^{2} B\left(K A_{1}^{*} \frac{\dot{h}}{B}+K^{2} A_{4}^{*} \frac{h}{B}\right)
\end{aligned}
$$

The residual sum of squares of Eq. (4a) is:

$$
\Delta^{2}=\sum_{i=1}^{N}\left[L_{i}-\rho U^{2} B\left(K H_{1}^{*} \frac{\dot{h}}{B}+K^{2} H_{4}^{*} \frac{h}{B}\right)\right]
$$

In order to minimize the residual sum of squares, the partial derivatives of Eq. (5) are satisfied according to the least square algorithm:

$$
\begin{aligned}
& \frac{\partial \Delta^{2}}{\partial H_{1}^{*}}=0 \\
& \frac{\partial \Delta^{2}}{\partial H_{4}^{*}}=0
\end{aligned}
$$

Thus, equations with unknown variables $H_{1}^{*}$ and $H_{4}^{*}$ can be obtained from Eqs. (6):

$$
\rho B^{2} \omega\left(\begin{array}{cc}
\sum_{i=1}^{N} h_{i}^{2} & \omega \sum_{i=1}^{N} h_{i} \dot{h}_{i} \\
\sum_{i=1}^{N} h_{i} \dot{h}_{i} & \omega \sum_{i=1}^{N} h_{i}^{2}
\end{array}\right)\left(\begin{array}{c}
H_{1}^{*} \\
H_{2}^{*}
\end{array}\right)=\left(\begin{array}{c}
\sum_{i=1}^{N} L_{i} \dot{h}_{i} \\
\sum_{i=1}^{N} L_{i} h_{i}
\end{array}\right)
$$

So, $H_{1}^{*}$ and $H_{4}^{*}$ can be acquired by solving Eq.(7). In the same way, the aerodynamic derivatives $A_{4}^{*}$ and $A_{4}^{*}$ can be obtained from Eq.(4b).

Similarly, the other four aerodynamic derivatives $\mathrm{H}_{2}^{*}, \mathrm{H}_{3}^{*}$, $A_{2}^{*}$ and $A_{3}^{*}$ can be obtained when the bridge section is forced to oscillate torsionally in a sinusoidal manner in the flow. The torsional oscillation is conducted by the following torsion driving signal in the present work

$\alpha(t)=\alpha_{0} \sin \omega t$ 
where $\alpha_{0}$ and $\omega$ are the displacement amplitude and the circular frequency of the oscillation, respectively .

\subsection{Governing Equations}

In bridge wind engineering, the flow around the bridge section is always deemed as the incompressible, viscous flow. This class of flow is governed by the continuity and momentum equations, that in two-dimensional form can be written as follows:

$$
\begin{aligned}
& \frac{\partial u_{i}}{\partial x_{i}}=0 \\
& \frac{\partial u_{i}}{\partial t}+u_{j} \frac{\partial u_{i}}{\partial x_{i}}=-\frac{\partial p}{\partial x_{i}}+\frac{\partial \tau_{i j}}{\partial u_{j}}
\end{aligned}
$$

where $x_{i}(\mathrm{i}=1,2)$ is the Cartesian coordinate, $t$ is time, $u_{i}$ is velocity component, $p$ is the kinetic pressure, and $\tau_{i j}$ is the stress tensor.

\subsection{DES Turbulence Model}

The stress tensor, $\tau_{i j}$ in Eq. (10), can be written as the sum of the viscous and Reynolds stress tensors:

$\tau_{i j}=\tau_{i j}^{L}+\tau_{i j}^{T}$

where the viscous part $\tau_{i j}^{L}$ is:

$$
\tau_{i j}^{L}=v\left(\frac{\partial u_{i}}{\partial x_{j}}+\frac{\partial u_{j}}{\partial x_{i}}\right)
$$

According to Boussinesq's assumption, the Reynolds stress tensor, $\tau_{i j}^{T}$ is given as:

$\tau_{i j}^{T}=v_{t}\left(\frac{\partial u_{i}}{\partial x_{j}}+\frac{\partial u_{j}}{\partial x_{i}}\right)-\frac{2}{3} k \delta_{i j}$

where $k$ is the turbulent kinetic energy, $v$ the laminar kinematic viscosity of the fluid, $v_{t}$ the turbulent eddy viscosity and $\delta_{i j}$ the Kroneker delta.

In S-A model, the turbulent viscosity $v_{t}$ can be defined as follows:

$v_{t}=\bar{v} f_{v 1}$

where $f_{v 1}=\frac{\chi^{3}}{\chi^{3}+C_{v_{1}}^{3}}, \chi=\frac{\bar{v}}{v}, \bar{v}$ is the working variable. The working variable $\bar{v}$ can be obtained by the following transport equation:

$$
\frac{D \bar{v}}{D t}=C_{b_{1}}\left(1-f_{t_{2}}\right) \bar{S} \bar{v}+\frac{1}{\sigma}\left[\nabla \cdot((v+\bar{v}) \nabla \bar{v})+C_{b_{2}}(\nabla \bar{v})^{2}\right]-\left[C_{w_{1}} f_{w}-\frac{C_{b_{1}}}{k^{2}} f_{t_{2}}\right]\left[\frac{\bar{v}}{d}\right]^{2}
$$

All the variables and constants in Eq. (15) are given as follows, $C_{b_{1}}=0.1355, \sigma=\frac{2}{3}, C_{b_{2}}=0.622, C_{v_{1}}=7.1$

$$
\begin{aligned}
& C_{w_{1}}=\frac{C_{b_{1}}}{k^{2}}+\frac{1+C_{b_{2}}}{\sigma}, f_{t_{2}}=C_{t_{3}} \exp \left(-C_{t_{4}} \chi^{2}\right) \\
& C_{t_{3}}=1.2, C_{t_{4}}=0.5, k=0.41, C_{w_{2}}=0.3, C_{w_{3}}=2.0 \\
& f_{w}=g\left[\frac{1+C_{w_{3}}^{6}}{g^{6}+C_{w_{3}}^{6}}\right]^{\frac{1}{6}}, g=r+C_{w_{2}}\left(r^{6}-r\right), r=\frac{\bar{v}}{\bar{S} k^{2} d^{2}} \\
& f_{v_{2}}=1-\frac{\chi}{1+\chi f_{v_{1}}}, f_{v_{1}}=\frac{\chi^{3}}{\chi^{3}+C_{v_{1}}^{3}}, \bar{S}=|\omega|+\frac{\bar{v}}{k^{2} d^{2}} f_{v_{2}}
\end{aligned}
$$

where $d$ is the distance from any point in the flow field to the nearest wall surface, $\omega$ the magnitude of the vorticity.

The DES turbulence model based on S-A model is obtained through replacing the distance to the nearest wall $d$ in Eq. (15), by $d^{2}$, which is defined as $d^{\prime}=\min \left(d, c_{D E S} \Delta\right)$. In which $\Delta$ is the largest distance from the center of the grid center to the adjacent cells, The model constant coefficient is taken as 0.65 . In the boundary layer near the wall, $\mathrm{d} \leq c_{D E S} \Delta$, the model degenerates into S-A model described by Eq. (15), and the model works in the RANS mode. When $\mathrm{d}>c_{D E S} \Delta$, the model works in Large Eddy Simulation mode.

\subsection{Dynamic Meshes for Numerical Simulation}

The bridge deck oscillations in the wind field can be operated through the moving boundary and dynamic mesh technique. In view of the arbitrary Lagrangian-Eulerian formulation, the integral form of the conservation equations for a generalized scalar, $\varphi$, on arbitrary control volumes with the moving boundary can be given as follows:

$$
\frac{d}{d t} \int_{V} \rho \varphi d V+\int_{\partial V} \rho \varphi\left(\stackrel{\mathrm{r}}{u}-\stackrel{\mathrm{r}}{u_{s}}\right) \cdot d A=\int_{\partial V}^{\mathrm{r}} \Gamma \nabla \varphi \cdot d A+\int_{V} S_{\varphi} d V
$$

where $V$ is the volume of the control volume, $\partial V$ the moving boundary of the control volume, $u$ flow velocity vector, $u$. velocity vector of the moving mesh, $\Gamma$ the diffusion coefficient, $S_{\varphi}$ the source term of the generalized scalar $\varphi$. The only moving boundary, considered in the present work is bridge deck surface, and it can be conducted using the driving signal defined as Eq.(2) or Eq.(8). This work is done by self-developed codes developed in the programming platform of Visual $\mathrm{C}++$. The mesh velocity $u_{s}$ in the moving mesh region can be easily obtained from the oscillations of the moving boundary.

The quality of the grid will become poor after every iteration time step. In order to adjust the size and shape of the grid, the spring smoothing method and the local remeshing method are utilized in this paper. The spring smoothing method can adjust the size and shape of the grid through the elastic deformation. When the displacement of boundary nodes are larger or less than the specified size, the local remeshing method will be activated to merger or split poor cell in this paper. 


\subsection{Solution Procedure}

Because the bridge section is deemed to be a rigid body in flow, one needs only to solve the governing equations among the fluid domain without concern for the aeroelastic response of the bridge. Eq. (16) is the integral form of the governing equations which considers the mesh velocity. In the equation, the first term on the left side is the unsteady term, the second on the right side is the convective term, and the first term is the diffusive term. The numerical solutions of Eq. (16) are computed using finite volume method. The second order upwind scheme for convective terms and the second order implicit scheme for unsteady terms are used. Diffusive terms are treated using a conventional central difference scheme. SIMPLE (Semi-Implicit Method for Pressure-Linked Equations) algorithm is used to achieve the pressure-velocity decoupling.

\section{NUMERICAL SIMULATIONS}

\subsection{Structure Geometry and Mesh Generation}

Selected test cases corresponding to turbulent flows around a thin flat plate and the girder section of Great Belt East Bridge are presented here. Their cross-sections are shown in Fig. (1). Section A represents the thin flat plate and section B is the Great Belt East Bridge suspended spans cross- section. Fig. (2) gives their computing grids.

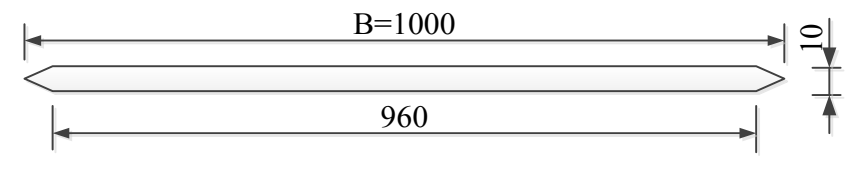

(a) The thin flat plate (Unit: $\mathrm{mm}$ )

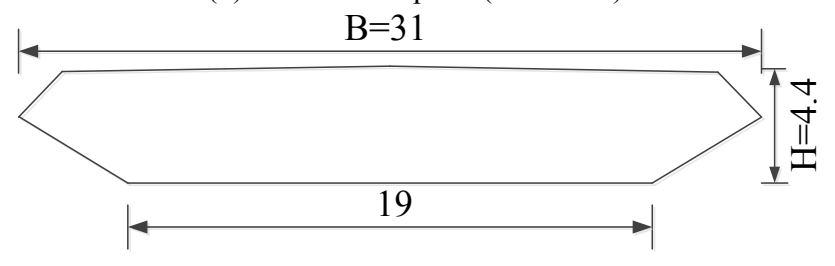

(b) Girder section of Great Belt East Bridge (Unit: m)

Fig. (1). Geometric model.

The thin flat plate and the girder section lengths are chosen as characteristic length and viewed as B, which is marked in Fig. (1). The computational domain is rectangular, and occupies a $16 \mathrm{~B} \times 20 \mathrm{~B}$ square in both cases, with the model section is located $8 \mathrm{~B}$ away from the inlet; and the distance from the upper or lower boundary to the center of the model section is also $8 \mathrm{~B}$. This arrangement can ensure the appropriateness of imposing the unperturbed free stream velocity at the inlet as well as the upper and lower boundaries of the domain. In the process of establishing the meshing, the inner boundary meshing is denser than that in exterior boundary to give consideration to computing power and computing precision. Meanwhile, the viscous boundary layer over the model surface is well resolved by the fine mesh with the overall y-plus less than 1, which can eliminate the need for a boundary layer treatment. At the inlet, the velocity entrance boundary is applied. The pressure outlet boundary is applied to the outlet. The no-slip boundary condition is imposed at the model surface as well as the upper and lower boundaries. The whole mesh region is defined as dynamic mesh region.

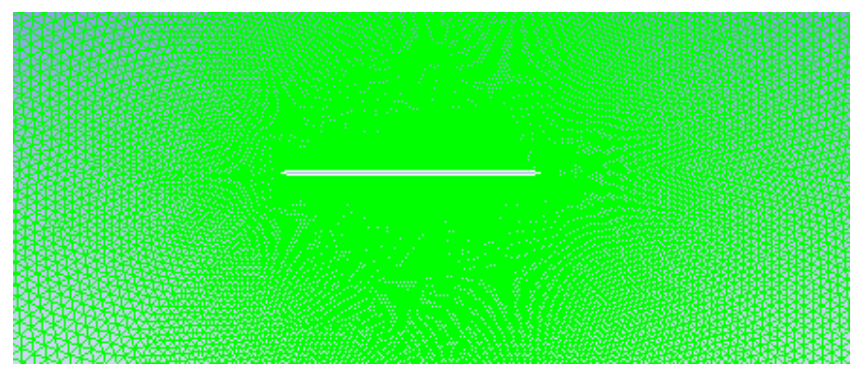

(a) The thin flat plate,

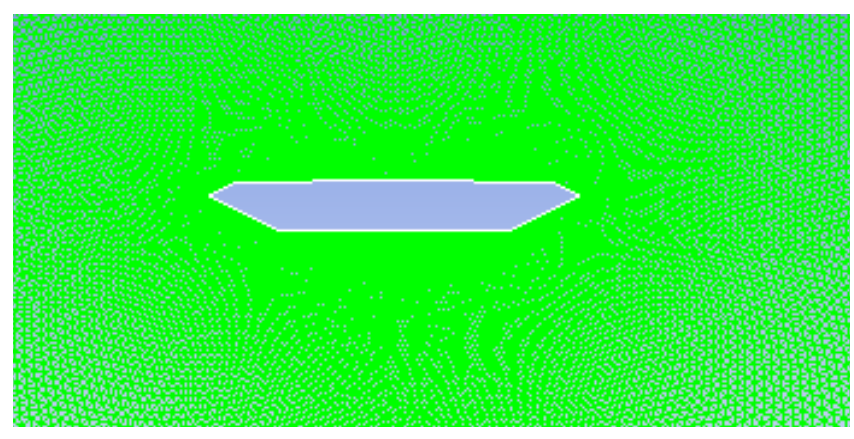

(b) Girder section of Great Belt East Bridge

Fig. (2). Model grids.

\subsection{Aerodynamic Force Coefficients and Discussion}

For the thin flat plate, forced motion simulations are conducted using the driving signals as follows: vertical signal $h=0.02 \sin (4 \pi t)$, torsion signal $\alpha=4 \sin (4 \pi t)$; and the simulations cover the wind velocity range from 4 to $40 \mathrm{~m} / \mathrm{s}$. For Great Belt East Bridge, the simulations are performed with the reduced wind velocities: $2.5,5,7.5$ and 10. The reduced wind flow velocity is given by $U / f B$, where $U$ is the inflow velocity, B is the bridge deck width and $f$ is the natural frequency of the structure. Vertical driving signal is $h=0.1 \sin (0.4 \pi t)$, and torsion driving signal is $\alpha=4 \sin (0.4 \pi t)$. These two examples only show computation of the aerodynamic derivative values for zero angle of attack.

The aerodynamic forces by simulation include the lift $\mathrm{L}(\mathrm{t})$ and the moment $\mathrm{M}(\mathrm{t})$. They are usually expressed in non-dimensional form as follows:

$C_{l}=\frac{L(t)}{0.5 \rho U^{2} B}, C_{m}=\frac{M(t)}{0.5 \rho U^{2} B^{2}}$,

where $C_{1}$ is the lift coefficient; $C_{m}$ is the moment coefficient; $\rho$ is the fluid density, taken as $1.225 \mathrm{~kg} / \mathrm{m}^{3} ; \mathrm{B}$ and $\mathrm{D}$ are the width and depth of the bridge deck, respectively; $U$ is the flow velocity.

Figs. (3 and 4) show the time history of the lift and moment coefficients when models $\mathrm{A}$ and $\mathrm{B}$ are forced in vertical motion, respectively. In order to carry out comparative analysis, the lift and moment coefficients acquired by RANS with RNG $k-\varepsilon$ turbulence model are also shown in Figs. (3 and 4). Comparing Fig. (3) with Fig. 
(4), it can be observed that the aerodynamic forces of model $\mathrm{B}$ have more distinct fluctuation than model A. One reason is that there are some more prominent internal disturbances in the flow fields for model B due to its irregular geometry. Another reason is that flow separation and vortex shedding in the flow fields are not quite obvious for model A owing to its very small thickness. As can be seen from Fig. (4), the aerodynamic lift and moment coefficients curves calculated by RANS look relatively smooth, while the results obtained by DES show obvious fluctuation. The reason for this phenomenon can be found in Fig. (5), which shows the instantaneous eddy-viscosity contours of model B in nearwake region, where vortex-shedding occurs. As expected, the RNG $k-\varepsilon$ turbulence model does not allow strong gradients and complex distribution of the turbulent viscosity in areas of massive separation, so much so that it is not able to sustain the vortex-shedding unsteadiness, even with very fine meshes. While DES turbulence model is capable of simulating the sophisticated distribution of the turbulent
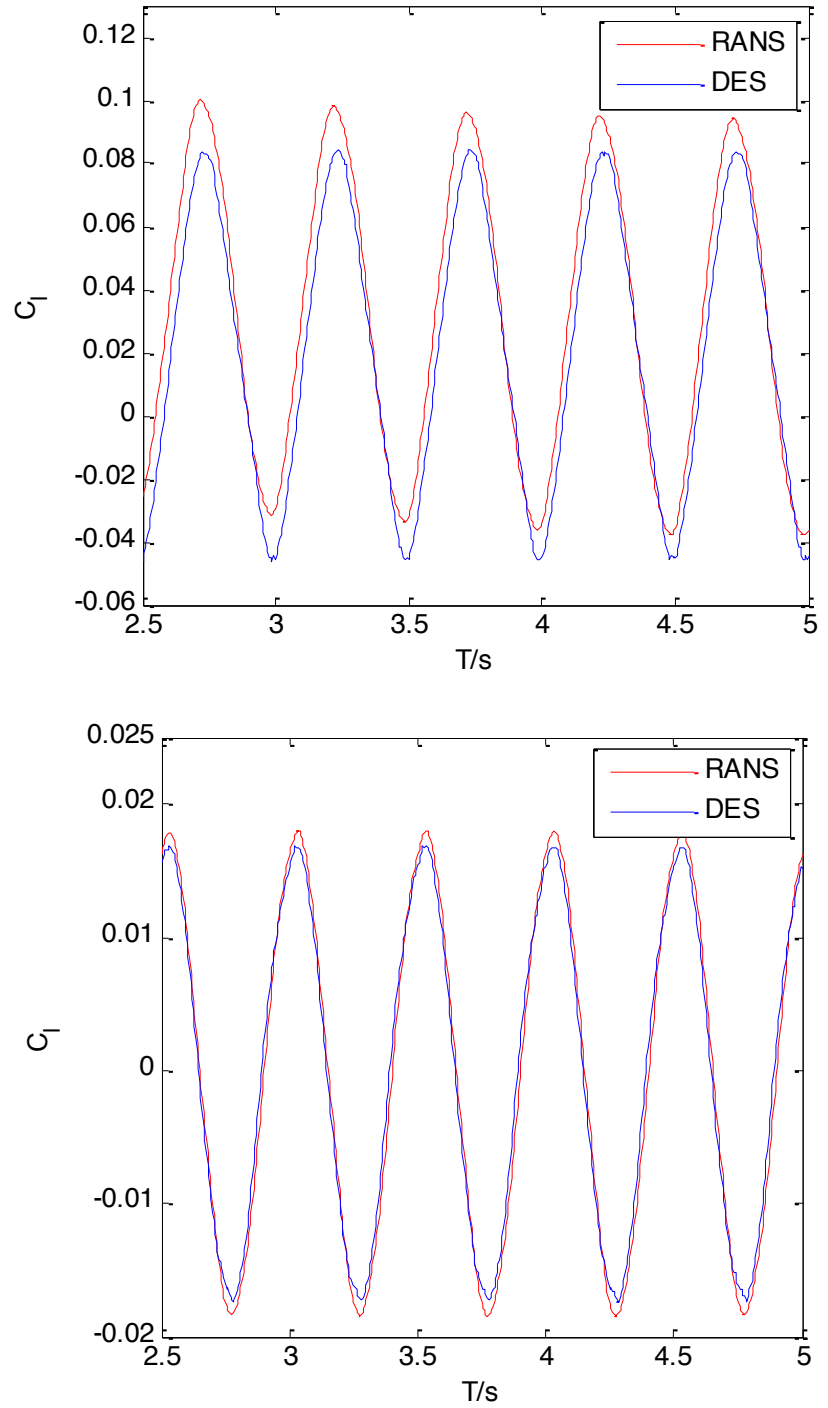

Fig. (3). Time histories of aerodynamic coefficients of model A undergoing vertical motion $(\mathrm{u}=16 \mathrm{~m} / \mathrm{s})$. viscosity. It means that the DES algorithm can capture the smaller vortexes, and reflect the complicated features of the flow around bridge decks. The discrepancy of the numerical results between DES and RANS for the aerodynamic coefficients of model B can be seen clearly from Fig. (4). Fig. (6) gives the reason for this discrepancy when the lift coefficient reach the maximum values. On the upper side of the bridge deck surface, the negative pressure region simulated from DES is larger than that from RANS. The reverse is true on the lower side of the bridge deck surface. This results in the higher lift coefficients obtained from DES than that from RANS. RANS creates relatively large local zones of low pressure acting on the front end of the lower surface and on the rear end of the upper surface. As a result, the DES results are larger than the RANS results for the moment coefficients.
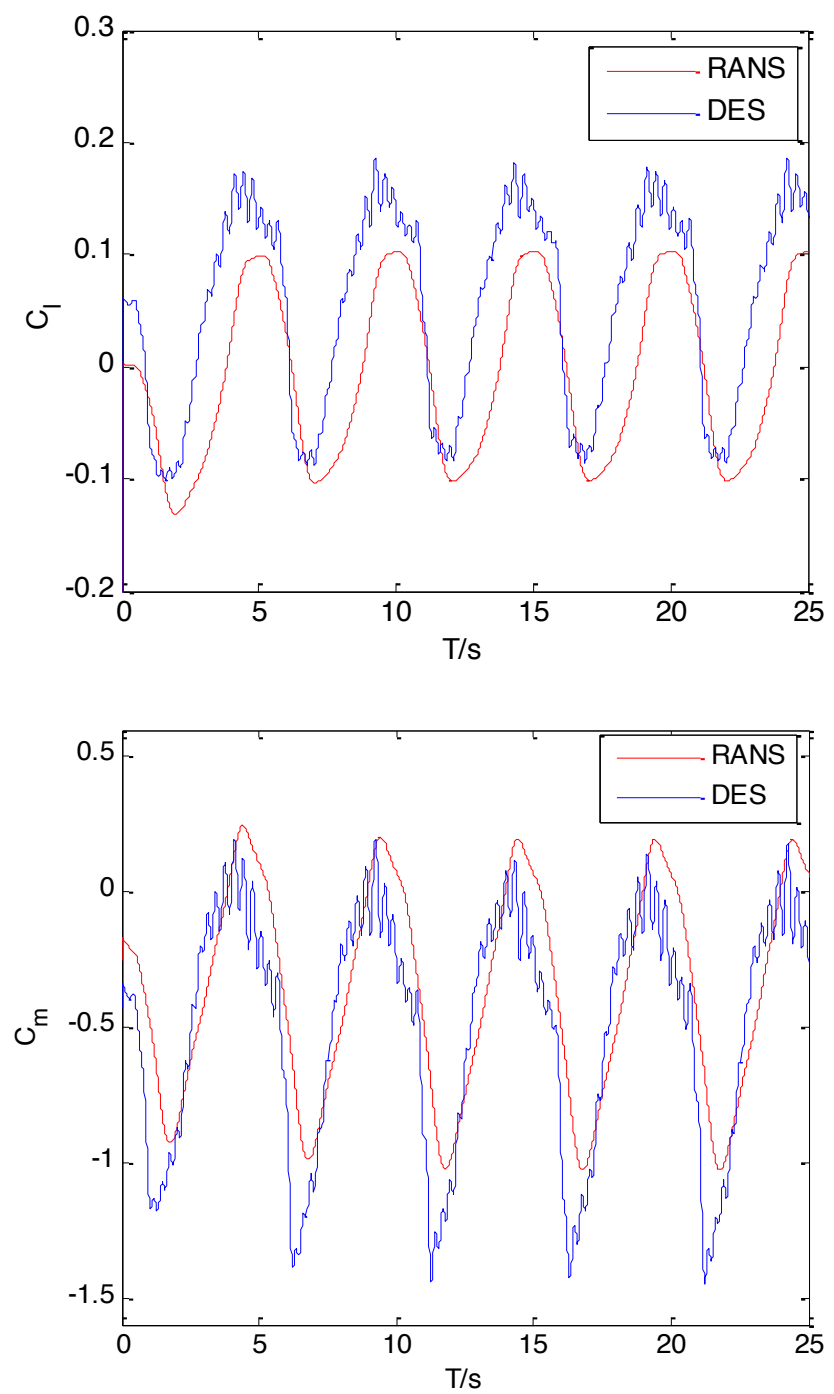

Fig. (4). Time histories of aerodynamic coefficients of model B undergoing vertical motion $(\mathrm{U}=31 \mathrm{~m} / \mathrm{s})$.

It is worth mentioning specially that the aerodynamic forces of model A and model B contain significant steady aerodynamic forces. Only by eliminating the impact of the 
steady aerodynamic forces, the aerodynamic lift and moment coefficients can be used to compute the aerodynamic derivatives.

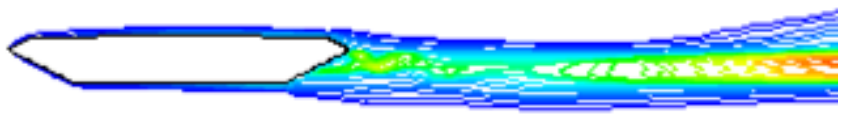

a)

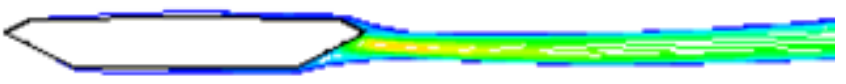

b)

Fig. (5). Computed eddy viscosity field around bridge deck corresponding to the instance of the maximum lift for model B undergoing vertical motion $(\mathrm{U}=31 \mathrm{~m} / \mathrm{s})$.

\subsection{Aerodynamic Derivatives and Discussion}

Once the aerodynamic forces are obtained, aerodynamic derivatives can be determined based on the least square algorithm described in the Section 2. The simulated aerodynamic derivatives of the thin flat plate are shown in Fig. (7), while Theodorsen 's theoretical solution [18] are also given for comparison. It can be found easily from Fig. (7) that the overall trend of the simulated results by DES meets well with the theoretical solution. Aerodynamic derivative values are in good agreement with the theoretical solution when the reduced wind velocities are small (less than $10 \mathrm{~m} / \mathrm{s}$ ). As the reduced wind velocity increases, some aerodynamic derivative values, such as $H_{2}^{*}$ and $A_{2}^{*}$, deviate slightly from the theoretical value. Nevertheless, the overall simulated results are encouraging. More importantly, it can be can seen that DES method has the better calculation precision than RANS, especially for $H_{4}^{*}$ and $A_{4}^{*}$.

Fig. (8) presents the computed values for Great Belt East Bridge, while the results calculated by virtue of DVM [15], LBM [19], RANS [20] and Poulsen's experimental data [21] are also given to demonstrate the usefulness and effectiveness of DES. Even though the wind tunnel test results are not complete, it is obvious that DES method is in better agreement with the wind tunnel test better than the other methods for $H^{*}, A^{*}$ and $A_{2}^{*}$. For $H^{*}$ and $H^{*}$, the DES results have the poor accuracy, but still conform to the change trend of the wind test results, and have almost the same accuracy as the RANS results. For $A_{3}^{*}, \mathrm{LBM}$ and DVM are in good agreement with the wind tunnel test, and are superior to DES and RANS. But DES still remains a distinct advantage over RANS. For $H_{3}^{*}$, at low reduced velocity, DES meet with the experimental values well, and is superior to other methods, while at high reduced velocity, its results are slightly less accurate than that of other methods. For $A_{4}^{*}$, DES has the same trend as RANS and DVM. The comparisons above justify that on the whole, the DES method adopted in this paper has obvious advantage over RANS and DVM in calculation precision, especially for some important aerodynamic parameters for the girder cross-sections, such as $H_{1}^{*}, H_{2}^{*}, H_{3}^{*}, A_{1}^{*}$, and $A_{2}^{*}$.

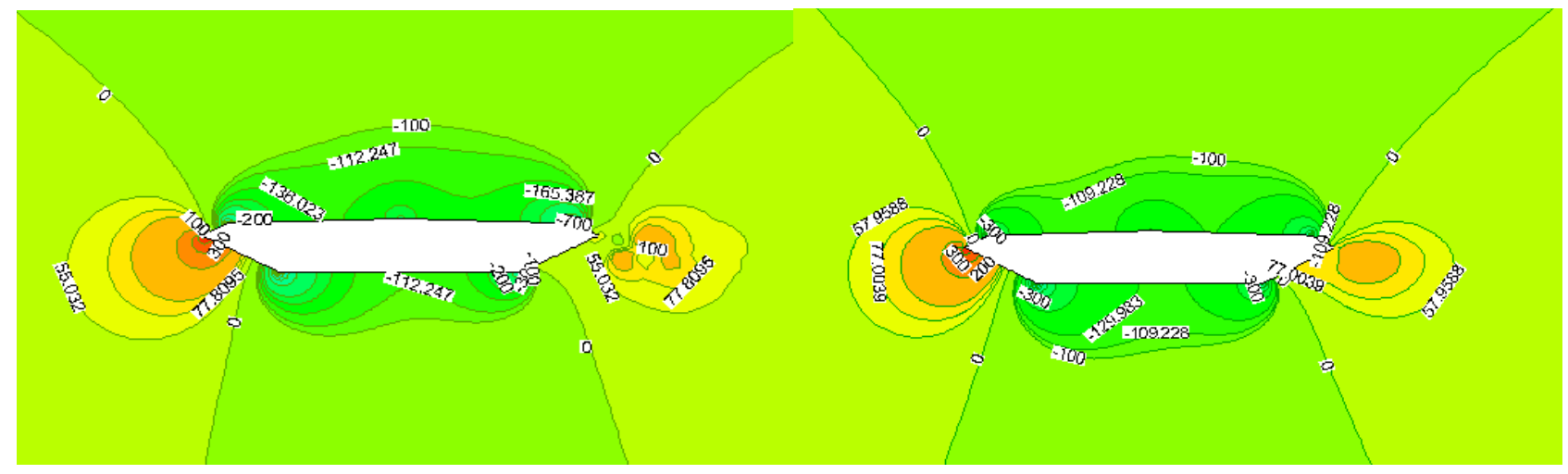

(a)DES

(b)RANS

Fig. (6). Pressure contours around bridge deck corresponding to the instance of the maximum lift for model B undergoing vertical motion $(\mathrm{U}=31 \mathrm{~m} / \mathrm{s})$
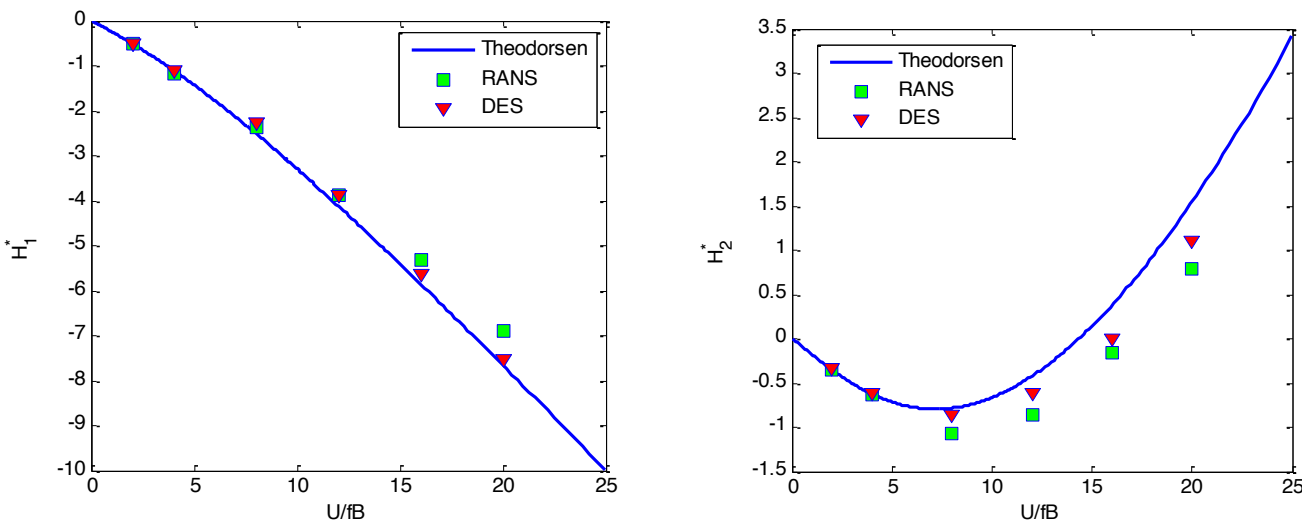

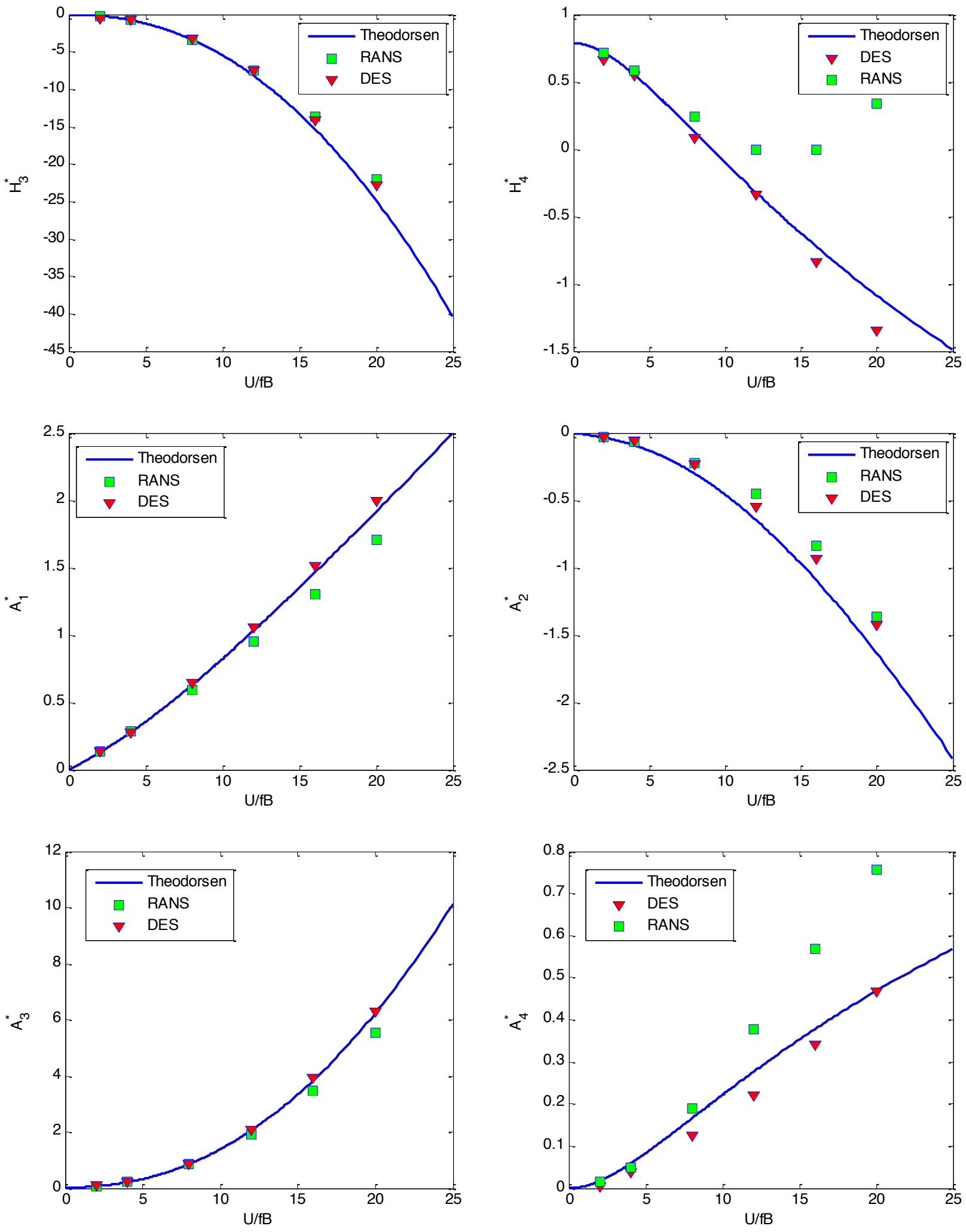

Fig. (7). Aerodynamic derivatives of thin flat plate. 

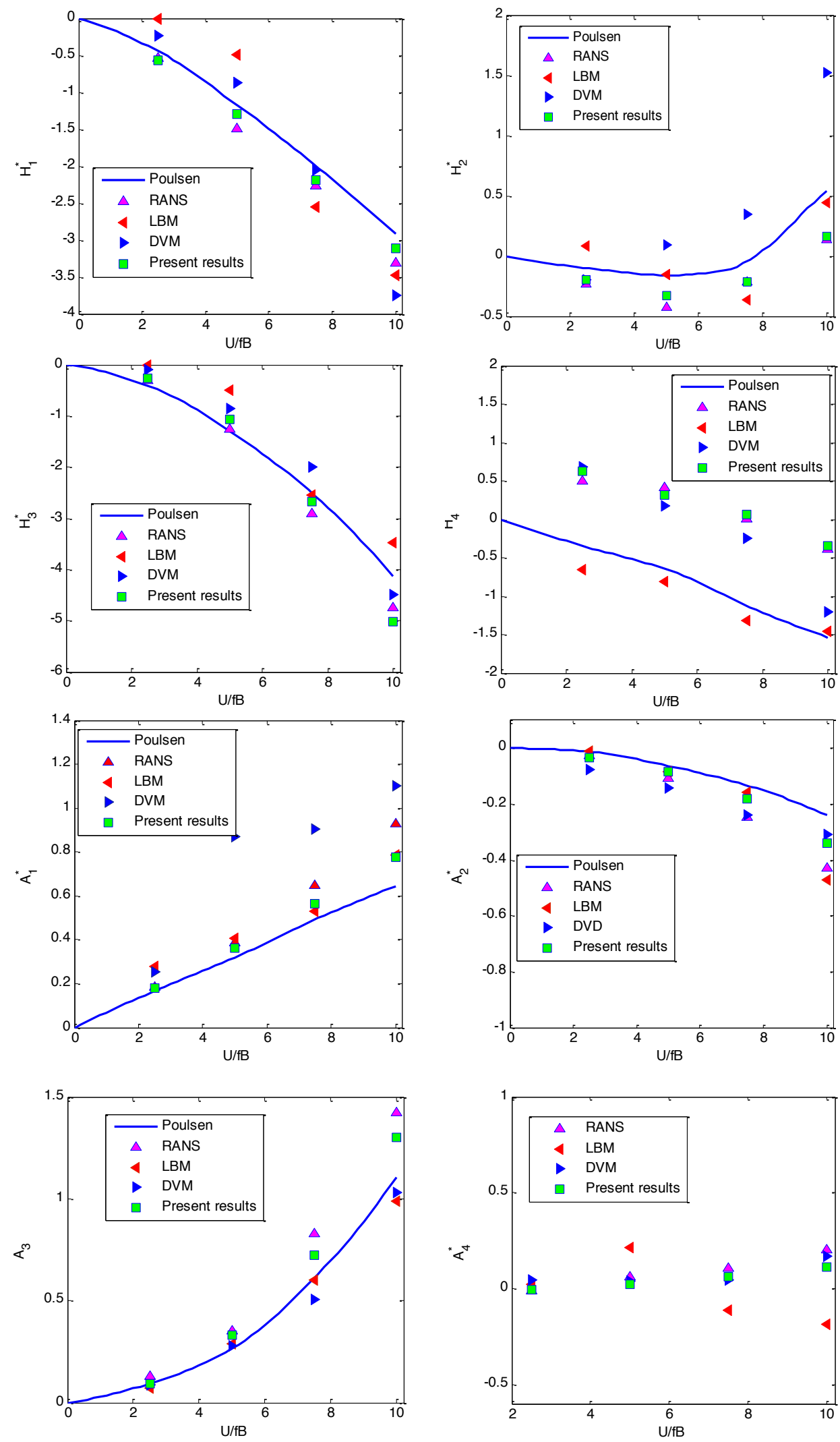

Fig. (8). Aerodynamic derivatives of great belt east bridge.

\section{CONCLUSION}

This paper aims to find a more accurate numerical method for the prediction of aerodynamic derivatives of the

bridge. First, the identification method for aerodynamic derivatives is elaborated. Then, the forced vibrations of the bridge decks in the flow fields are successfully achieved by self-developed codes combined with FLUENT software 
employing DES turbulence model, thereby the aerodynamic forces can be computed. At the end, the reliability of DES turbulence model for the prediction of aerodynamic derivatives of the bridge is discussed through numerical examples.

As an example, the thin flat plate and the Great Belt East Bridge suspended spans cross-section are investigated to calculate their aerodynamic force coefficients and aerodynamic derivatives through applying this method. For the thin flat plate, the simulation values of the aerodynamic derivatives are in good agreement with the theoretical curves. For the box section (Great Belt East Bridge), the DES results for the box section at low reduced velocity are more close to the experimental values, while at high reduced velocity, the discrepancy between the DES results and wind test values becomes obvious. Comparing with the other numerical methods, DES method has the better calculation precision than RANS, and is superior to DVM for most of aerodynamic derivatives. These comparisons are powerful evidence of the reliability and applicability of the present CFD method. Numerical examples also prove that DES has a distinct advantage over RANS in simulating flow separation, reattachment and vortex shedding unsteadiness, which are the salient features for the flow field around the bridge. Thus, the DES method is more worthy of being popularized and is applied to obtain aerodynamic derivatives of bridge decks.

\section{CONFLICT OF INTEREST}

The authors confirm that this article content has no conflict of interest.

\section{ACKNOWLEDGEMENTS}

The research was supported by International Cooperative Program (Funding Codes: 2011DFA21460).

\section{REFERENCES}

[1] J.H. Walther, and A. Larsen, "Two dimensional discrete vortex method for application to bluff body aerodynamics," J. Wind Eng. Indust. Aerodyn., vol. 67-68, pp. 183-193, 1997.

[2] J.H. Walther, and A. Larsen, "Aeroelastic analysis of bridge girder sections based on discrete vortex simulations," J. Wind Eng. Indust. Aerodyn., vol. 67-68, pp. 253-265, 1997

[3] J. H. Walther, and A. Larsen, "Discrete vortex simulation of flow around five generic bridge deck sections," J. Wind Eng. Indust. Aerodyn., vol. 77-78, pp. 591-602, 1998.
[4] R. P, Silva, M. J. Tarini, and A. Larsen, "Computer modelling of flow around bridges using LES and FEM," J. Wind Eng. Indust. Aerodyn., vol. 77-78, pp. 643-651, 1998.

[5] J. B. Frandsen, "Numerical bridge deck studies using finite elements.Part I: flutter," J. Fluids Struct., vol. 19, pp. 171-191, 2004.

[6] J. B. Frandsen, "Computational Fluid-Structure Interaction Applied to Long-Span Bridge Design," Cambridge University, 1999.

[7] M. Sarwar, T. Ishihara, K. Shimada, Y. Yamasakic, and T. Ikedad, "Prediction of aerodynamic characteristics of a box girder bridge section using the LES turbulence model," J. Wind Eng. Indust. Aerodyn., vol.10-11, pp. 1895-1911, 2008.

[8] L. Huang, H. 1. Liao, B. Wang, and Y. 1. Li, "Numerical simulation for aerodynamic derivatives of bridge deck," Simulation Modelling Practice and Theory, vol.17, pp. 719-729, 2008.

[9] A .L. Braun, and A. M. Awruch, "Finite element simulation of the wind action over bridge sectional models: Application to the Guamá River Bridge (Pará State, Brazil) ,"Finite Elements in Analysis and Design, vol. 44, pp. 105-122, 2008.

[10] F. C. Cao, H. F. Xiang, and A. R. Chen, "Numerical assessment of aerodynamic derivatives of thin flat plate," J. Tongji Univ., vol. 27, pp. 131-135, 1999.

[11] F. C. Cao, H. F. Xiang, and A. R. Chen, "Numerical assessment of aerodynamic derivatives and critical wind speed of flutter of bridge decks," ACTA Aerodynamic Sinica, vol. 18, pp.26-33, 2000.

[12] Z.W. Zhu, Z.Q. Chen, and W. F. Chen, "Assessment of aerodynamic derivatives of the ideal flat plate based on the moving grid method," J. National Univ. Defense Tech., vol. 24, pp. 13-17, 2002.

[13] Z.W. Zhu, and Z.Q. Chen, "Numerical simulations for aerodynamic derivatives and critical flutter velocity of bridge deck," China J. Highway and Transp., vol. 17, pp. 41-45, 2004.

[14] Z.W. Zhu, Z.Q. Chen, and M. Gu, "Evaluating flutter derivatives of the thin plate by applying the numerical simulation method," $J$. Hunan Univ. (Natural Sciences), vol. 32, pp. 11-15, 2005.

[15] Z. Y. Zhou, A.R. Chen, and H.F. Xiang, "Numerical assessment of aerodynamic derivatives and critical wind speed of flutter of bridge decks by discrete vortex method," J. Vib. Eng., vol. 32, pp. 327$331,2002$.

[16] J. Y. Tu, G. H. Yeoh, and C. Q. Liu, Computational Fluid Dynamics: A Practical Approach, Elsevier Press, 2008, pp. 132147.

[17] P. R. Spalart, and S. R. Allmaras, "A one-equation turbulence model for aerodynamic flows,"AIAA-92-0439, 1994.

[18] T. Theodorsen, "General theory of aerodynamic instability and mechanism of flutter," NACA Report No. 496, 1935.

[19] T. C. Liu, "Lattice Boltzmann Method used for bridge structure aeroelastic numerical calculation," Tongji University, 2007.

[20] Y. J. Ge, and H. F. Xiang,"Computational models and methods for the aerodynamic stability of long-span bridges," China Civil Eng. $J$. , vol. 41, pp. 86-93, 2008.

[21] N.K. Poulsen, A. Damsgaard, T. A. Reinhold, "Determination of flutter derivatives for the Great Belt Bridge," J. Wind Eng. Ind. Aerod, vol. 41, pp. 153-164, Oct. 1992. 